\title{
Infant Exposure to Bisphenol A Can Be Quantitatively Assessed by a Simply Improved High-Performance Liquid Chromatography- Tandem Mass-Spectrometry Method
}

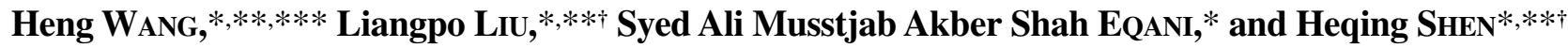 \\ *Key Laboratory of Urban Environment and Health, Institute of Urban Environment, Chinese Academy of \\ Sciences, Xiamen 361021, China \\ **University of Chinese Academy of Sciences, Beijing 100049, China \\ ***Key Laboratory of Health Risk Factors for Seafood of Zhejiang Province, Zhoushan Municipal Center for \\ Disease Control and Prevention, Zhejiang, Zhoushan 316021, China
}

\begin{abstract}
To quantitatively measure trace levels of bisphenol A (BPA) in infant urine, a simply improved high-performance liquid chromatography (HPLC)-tandem mass-spectrometry method was developed and validated. In the present work, a ZORBAX SB-C ${ }_{18}$ column $(2.1 \times 30 \mathrm{~mm}, 3.5 \mu \mathrm{m})$ was used for trapping and isolating BPA from the HPLC mobile phase. The result showed that the trapping column can provide effective separation from the background BPA to the sample BPA. This method has a limit of detection (LOD) of $0.048 \mu \mathrm{g} / \mathrm{L}$, spiked recoveries $85.4 \%(0.50-10.0 \mu \mathrm{g} / \mathrm{L})$, and relative standard deviations of $1.8-15.9 \%$. By using its low-LOD advantage, we firstly reported that Chinese infants $(n=48$ with age $<6$ months) have been ubiquitously exposed to BPA (detection frequency of 93\%) with an median level of $0.13 \mu \mathrm{g} / \mathrm{L}$ (ranging from below LOD to $5.04 \mu \mathrm{g} / \mathrm{L}$ ).
\end{abstract}

Keywords Infant urine, trace level BPA, trapping column, HPLC-MS/MS

(Received October 17, 2016; Accepted January 30, 2017; Published July 10, 2017)

\section{Introduction}

Bisphenol A is used to produce polycarbonate plastics and epoxy resins; the latter has been commonly used to line metal cans for tightly containing beverage and preserved food, including baby food. ${ }^{1-3}$ With a worldwide annual production estimated to be more than 3.5 million tons, BPA has shown endocrine-disrupting effects and potential adverse health effects. ${ }^{4,5}$ These may cause severe environmental health problems and have thus triggered the increasing public concerns. . $, 3,6-10$ Many surveys have been conducted to monitor human exposure to BPA, especially concerning the adults and old children ( $>6$ years old). The methods adopted in the surveys have usually included solid-phase extraction (SPE), and/or liquid-liquid extraction, as well as subsequent high-performance liquid chromatography tandem mass-spectrometry (HPLC-MS/ MS) quantification. ${ }^{11-16}$ For example, the National Health and Nutrition Examination Survey (NHANES) 2015 reported that more than $90 \%$ of urine samples from participants $>6$ years old can detect BPA with a median of $2.7 \mu \mathrm{g} / \mathrm{L}$ in $2003-2004$ and $1.4 \mu \mathrm{g} / \mathrm{L}$ in $2011-2012 .{ }^{17}$ However, only a few reports have shown that infant cohorts were exposed to BPA. For example, one study reported that the investigated infant (3-15 months old) urine samples had a mean of $0.96 \mu \mathrm{g} / \mathrm{L} \mathrm{BPA},{ }^{18}$ few other reports ${ }^{19-22}$ also showed that the newborn and/or young infant

† To whom correspondence should be addressed. E-mail: lpliu@iue.ac.cn (L. L.); hqshen@iue.ac.cn (H. S.) urine indicated trace levels of BPA being detected. These limited data revealed that the BPA levels in the newborns and infants may be lower than the levels in the old children and adults. To address the potential risk of BPA existing in these susceptible cohorts, its reliable measurement in urine with a limitof-detection (LOD) down to one part per trillion (ppt) is usually required. Therefore, any background contamination from water, organic solvents, and/or leaching from plastic pipes ${ }^{23,24}$ should be determined in order to ensure the quality of acquired data.

To eliminate or minimize any background contamination, various techniques have been tried. Völkel ${ }^{20}$ had noticed that there was a BPA peak with a signal-to-noise $(S / N)$ of 10 when a calibration standard without BPA was injected. To offset the interference, the method LOQ had to elevate to a high level $0.45 \mu \mathrm{g} / \mathrm{L}$ by background substraction. Some other studies $^{21-23,25,26}$ using the dansyl derivation to avoid any background contamination from the mobile phase or the postsample-preparation steps. Although this technique can greatly enhance the method sensitivity, its drawback is also apparent due to extra, tedious derivatization steps. In another way, using HPLC grade water ${ }^{18}$ is an expensive choice for reduced any mobile-phase contamination.

Inspired by the fact that a trapping column can isolate background perfluorinated compounds contamination, ${ }^{27}$ we designed subsequent work to develop a simple and efficient method to resolve the problem of background BPA contamination. With the improved method, we successfully measured tracelevel BPA in infant urine samples, and found that the investigated Chinese infants were widely exposed to BPA. 
Table 1 Parameters for MRM acquisition of the analytes

\begin{tabular}{lccccccc}
\hline Analyte & Detection mode & Precursor ion $(m / z)$ & S-lens/Hz & SRM1 $(m / z)$ & Collision energy/eV & SRM2 $(m / z)$ & Collision energy/eV \\
\hline BPA & - & 227 & 71 & 133 & 33 & 212 & 32 \\
BPA- ${ }^{13} C_{12}$ & - & 239 & 84 & 223 & 31 & 224 & 20 \\
\hline
\end{tabular}

a. Quantitative ions.

\section{Experimental}

\section{Reagents and materials}

The BPA standard was purchased from Aldrich-Sigma (purity $>98 \%$, St. Louis, MO, USA). BPA- ${ }^{13} \mathrm{C}_{12}$ (Cambridge Isotope Laboratories Ins, USA), HPLC-grade methanol, ethyl acetate and ammonium acetate (CNW Technologies) were purchased from Shanghai ANPEL Scientific Instrument Co., Ltd. $\beta$-Glucuronidase from Helix pomatia (Type HP-2, aqueous solution, $\beta$-glucuronidase activity $\geq 100000$ units $/ \mathrm{mL}$, sulfatase activity $\leq 7500$ units $/ \mathrm{mL}$ ) was purchased from Sigma-Aldrich. Deionized water for the preparation of buffers was purified by a Milli-Q ${ }^{\circledR}$ water-purification system (Millipore, Bedford, MA, USA). An Oasis HLB cartridge (Waters, $200 \mathrm{mg} / 6 \mathrm{~cm}^{3}$, Milford, MA, USA) was purchased from Waters.

Stock solutions of BPA and ${ }^{13} \mathrm{C}_{12}$-BPA were prepared at a concentration of $1 \mathrm{mg} / \mathrm{mL}$ in methanol. All stock solutions were stored at $-20^{\circ} \mathrm{C}$. Calibration standards, ranging from 0.20 to $25 \mu \mathrm{g} / \mathrm{L}$, were prepared from the stock solution through serial dilution by using a methanol-water mixture $(50 / 50, \mathrm{v} / \mathrm{v})$; isotope stock solutions $\left({ }^{13} \mathrm{C}_{12}\right.$-BPA, $\left.100 \mu \mathrm{g} / \mathrm{L}\right)$ were also prepared by using the same methanol-water mixture.

\section{Instrument}

The LC system consisted of a Thermo Fisher Scientific (San José, USA) Accela UHPLC pumping system, coupled with an Accela Autosampler and Degasser. Chromatographic separation was achieved via reversed-phase chromatography and gradient elution. Separations of the compounds were carried out on a Hypersil Gold aQ $\mathrm{C}_{18}$ column $(1.9 \mu \mathrm{m}, 100 \times 2.1 \mathrm{~mm}$, Thermo Fisher Scientific), kept at $30^{\circ} \mathrm{C}$. A ZORBAX SB-C $\mathrm{C}_{18}$ column $(2.1 \times 30 \mathrm{~mm}, 3.5 \mu \mathrm{m})$ was used as a trapping column. The mobile phase of water and methanol, was pumped at a flow rate of $0.3 \mathrm{~mL} / \mathrm{min}$. Optimized separation of BPA was obtained by using a linear gradient starting with a mixture of water (A) and methanol (B) solvents: 20\% B for $1 \mathrm{~min}$, followed by a linear gradient to $100 \% \mathrm{~B}$ within $7 \mathrm{~min}$. The solvent composition was then kept at $100 \%$ B for $2 \mathrm{~min}$, and finally the column was re-equilibrated at $20 \% \mathrm{~B}$ for $5 \mathrm{~min}$. The total run could be accomplished in $15 \mathrm{~min}$.

Analysis was performed on a triple quadrupole mass analyzer (TSQ Vantage, Thermo Fisher Scientific, San José, USA), fitted with a heated electrospray ionization (HESI) source operating in the negative-ion mode. The following working conditions were applied: spray voltage of $2.5(-) \mathrm{kV}$; vaporizer and capillary temperatures of 450 and $300^{\circ} \mathrm{C}$, respectively; sheath and auxiliary gas at 40 and 20 arbitrary units (a.u.), respectively; cycle time of $0.8 \mathrm{~s}$. The argon pressure in the collision cell (Q2) was set at 1.5 mTorr, and the mass resolution at the first (Q1) and third (Q3) quadrupole were set at $0.7 \mathrm{Da}$ at the full width at half maximum (FWHM). The precursor ion, S-lens RF amplitude, and collision energy (CE) in Q2 were optimized individually per compound and/or transition (Table 1). Quantification and confirmation data were acquired in a selected reaction monitoring (SRM) mode; the transitions are displayed as Table 1. Instrument control and data processing were carried out by means of Xcalibur Software 2.2 SP1.48 (Thermo Electron, San José, USA).

\section{Sample preparation}

An enzyme solution was prepared by diluting $20 \mu \mathrm{L}$ $\beta$-glucuronidase Type HP-2 from Helix pomatia with $10 \mathrm{~mL}$ of an ammonium acetate buffer $(0.77 \mathrm{~g}$ of ammonium acetate dissolved in $10 \mathrm{~mL}$ of Milli- $\mathrm{Q}^{\circledR}$ water, $\left.1 \mathrm{~mol} / \mathrm{L}\right)$. Each sample of $0.5 \mathrm{~mL}$ urine was diluted with $2 \mathrm{~mL}$ of the enzyme solution. Then, $50 \mu \mathrm{L}$ of the internal standard solution, containing ${ }^{13} \mathrm{C}_{12}$ BPA $(100 \mu \mathrm{g} / \mathrm{L})$, was also added, the samples were incubated subsequently at $37^{\circ} \mathrm{C}$ overnight under gentle agitation. The digested samples were extracted by adding $2.5 \mathrm{~mL}$ of ethyl acetate, and then vortexed vigorously for $10 \mathrm{~s}$ in a glass tube. The extraction was repeated 3 times. Afterwards phase separation was achieved by centrifugation at $1500 \mathrm{r} / \mathrm{min}$ for $10 \mathrm{~min}$. The ethyl acetate phase was transferred to another glass tube with a pasteur pipette. The extract was mixed and washed with $5 \mathrm{~mL}$ of water to decrease any inference of inorganic salt (ammonium acetate); the extracts were then evaporated under a gentle stream of nitrogen gas at $40^{\circ} \mathrm{C}$. The residue was reconstituted with $0.5 \mathrm{~mL}$ of methanol/water $(50 / 50$, $\mathrm{v} / \mathrm{v}$ ) by being vortexed vigorously for $15 \mathrm{~s}$, and then transferred into a HPLC vial.

\section{Method evaluation and quality control}

In order to evaluate this new methodology, a validation study was also conducted. To evaluate the linearity, BPA standards were prepared for the following concentrations i.e., 0, 0.40, 2.0, 10,20 and $50 \mu \mathrm{g} / \mathrm{L}$ in water/methanol (50/50, v/v). Afterwards, these standards were mixed at a 1:1 ratio, an internal standard of $20.0 \mu \mathrm{g} / \mathrm{L}{ }^{13} \mathrm{C}_{12}-\mathrm{BPA}$, resulting in final calibration concentrations of $0,0.20,1.0,5.0,10$ and $25 \mu \mathrm{g} / \mathrm{L}$ and $10 \mu \mathrm{g} / \mathrm{L}$ of the internal standards. Calibration curves were obtained from the ratios of the analyte to the internal-standard peak area, using linear regression with $1 / x$ weighting. The method was validated according to the FDA guidance for industry bioanalytical method validation. ${ }^{28}$ The limit of detection (LOD) and the limit of quantification (LOQ) were calculated based on the most intense transition. The LOD was calculated to be $3 S_{0}$, where $S_{0}$ is the standard deviation as the concentration approaches zero. $S_{0}$ was determined from six repeated measurements of low-level standards. The LOQ for $0.5 \mathrm{~mL}$ urine was defined as 3.33 times of the LOD. To validate the accuracy and precision of this method, six repeated analysis of spiked urine sample matrix at three levels (i.e. $0.50,1.0$ and $5.0 \mu \mathrm{g} / \mathrm{L}$ ) were performed; the accuracy was expressed as the percentage of the expected values, and the precision was expressed as the coefficient of variance $(\mathrm{CV} \%)$. A method blank was prepared from a mixture of 15 infant urine samples by stirring with $1 \%(\mathrm{~m} / \mathrm{m})$ activated charcoal overnight. ${ }^{29}$ Moreover, in order to evaluate the carryover effect of the high-concentration sample to the next sample in the analysis sequence, we tested three (3) blank samples after the highest sample of the standard curve. Finally, to qualify as a valid run, three method blanks, three quality- 


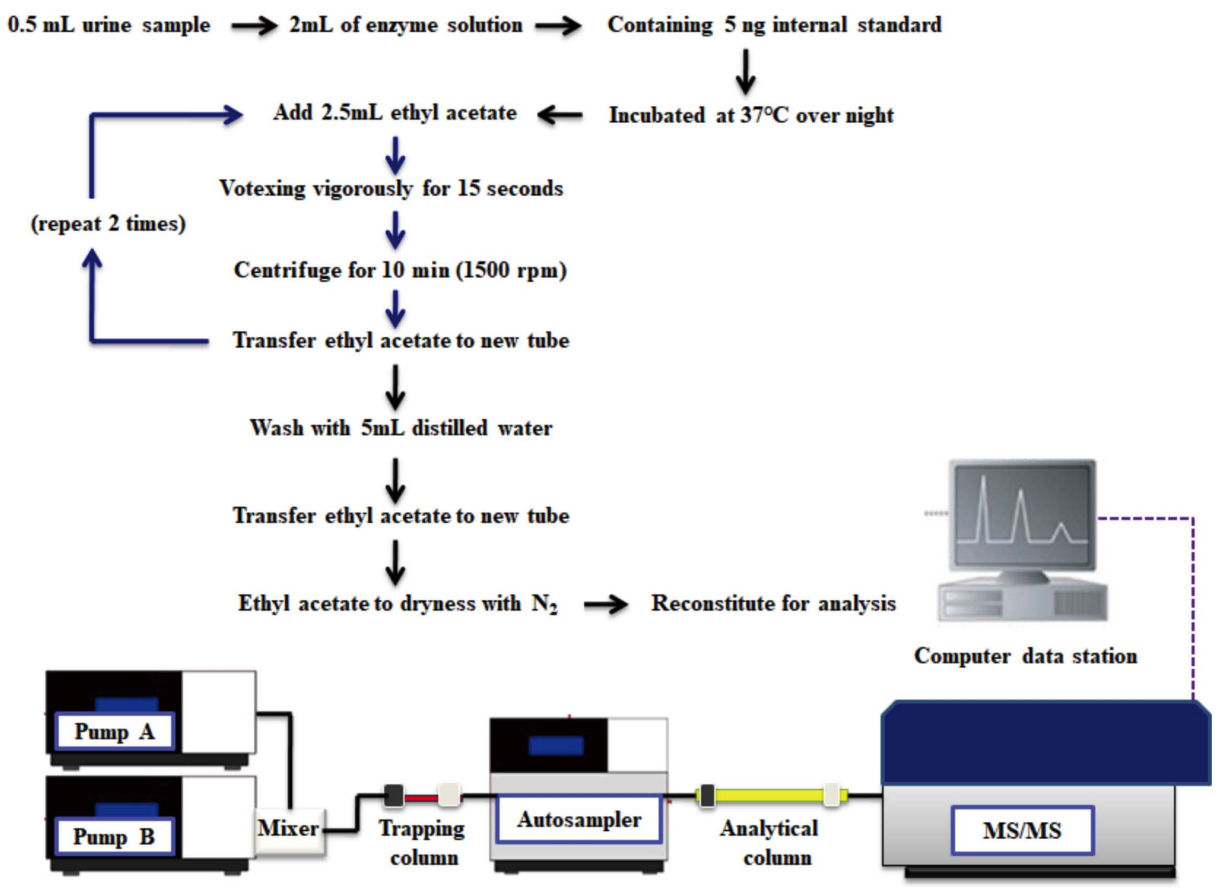

Fig. 1 Sample-preparation procedure and connection diagram for an on-line background cleanup UHPLC-MS/MS system.

control samples (sample matrix spiked with the BPA at 0.50 and $5.0 \mu \mathrm{g} / \mathrm{L}$ ), and several repeats of the $1.0 \mu \mathrm{g} / \mathrm{L}$ standard were inserted in each analytical batch (one repeat for every ten samples, approximately). The method blanks needed to have no contamination, completely. The analytical values of the qualitycontrol samples needed to be within $\pm 20 \%$ of the expected value. ${ }^{30}$

\section{Method application}

This method was used to analyze BPA from 48 infant urine samples. Urine samples were collected as part of an ongoing study of Maternal and Infant Population Exposure to Typical Environmental Pollutants and Reproductive Health conducted by Zhoushan Municipal Center for Disease Control and Prevention (Zhoushan, Zhejiang Province, China) and Institute of Urban Environment, Chinese Academy of Sciences (Xiamen, Fujian Province, China). Maternal and infants were recruited from Daishan Maternal and Child Health and Family Planning Service Center (Zhoushan, Zhejiang Province, China) from March 2012 to December 2014; the study was approved by the local ethical committee. Before sample collection, informed written consent was obtained from the parents to collect their infant's urine. The infants were aged from newborn to six months. The urine samples (disposable diaper) were collected at the time of each infant physical examination. The disposable diaper was stored at $-20^{\circ} \mathrm{C}$ immediately after collection until delivery to Zhoushan Municipal Center for Disease Control and Prevention, where the analysis was performed. Before analysis, all infant urine samples were released from each disposable diaper gel according to a previously published method. ${ }^{29}$

\section{Results and Discussion}

\section{Isolation of background BPA in mobile phase}

The main purpose of this study was to isolate any background
BPA from each sample BPA, and to obtain its urinary level for reliable exposure assessment. Initial experiments showed that the major background contaminations occurred during instrument analysis, demonstrated as a BPA peak in the chromatogram, even when nothing was injected into the instrument. BPA residual in the mobile phase had been speculated to be sources of contamination. ${ }^{20,31}$ Our tests showed that the background levels of BPA in the water and methanol fractions were at 0.05 and $0.0016 \mu \mathrm{g} / \mathrm{L}$, respectively. Before each sample injection, the BPA residuals in the mobile phase ( $80 \%$ water and $20 \%$ methanol) could be concentrated at the head of the analytical column during a period of $15 \mathrm{~min}$ equilibration; when the ratio of the methanol in the mobile phase gradually increased after sample injection, the concentrated BPA could be eluted as a peak that can just interfere with the targeting BPA peak severely. In order to isolate any interference, a trapping column (ZORBAX SB- $\mathrm{C}_{18}$ column, $2.1 \times 30 \mathrm{~mm}, 3.5 \mu \mathrm{m}$ ) was inserted in between the mixer and the injector; then, any BPA contamination from mobile phase was accumulated on the trapping column and later eluted when the methanol percentage was increased (Fig. 1). By adjusting the gradient program, the interfering BPA peak could be eluted from the analytical column with a retention time different from that of the targeting BPA peak (approximately 0.5 min delay, i.e., $t_{\mathrm{R}}=7.35$ and $7.81 \mathrm{~min}$, respectively, in panel $\mathrm{g}$ and $\mathrm{h}$ of Fig. 2). Hence, any potential BPA interference from mobile phase was completely avoided.

Two further experiments were conducted to verify the origin of these two BPA peaks (Fig. 2). In the first experiment, different concentrations of BPA standard solutions $(0,0.20,1.0$, $5.0,10,25 \mu \mathrm{g} / \mathrm{L})$ were analyzed. We noticed that the area of the first BPA peak increased proportionally, while the area of the second peak remained constant. In the second experiment, different column equilibration times (such as $0,10,20,40,60$ min) were set, and then we found that the areas of the second peak increased linearly to response the time setting. 
The equilibration time versus the peak area showed good linearity with equation of $y=751.16 x+6842.7\left(r^{2}=0.993\right)$. Therefore, we concluded that the interfering BPA peaks did come from the mobile phase.

As had been mentioned before, the BPA residual in water might be the major contributor to the second BPA peak. To confirm this, we purified some water by using a HLB cartridge. When the purified water was used as the mobile phase, the second BPA peak was disappeared, and only a very slight rising of baseline was observed. Because further water preparation is

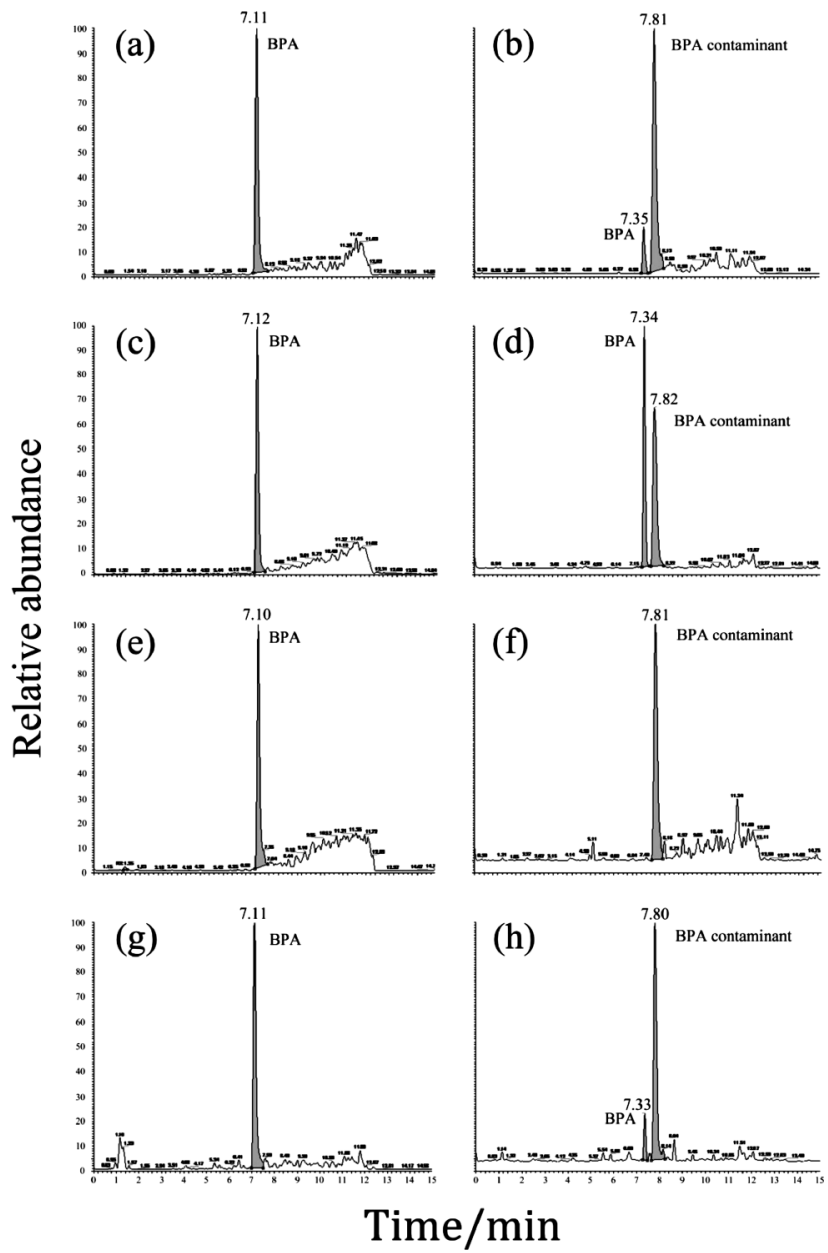

Fig. 2 Optimal representative chromatograms: (a and b) BPA of $0.2 \mu \mathrm{g} / \mathrm{L}$ standard before and after the trapping column insert; (c and d) BPA of $1.0 \mu \mathrm{g} / \mathrm{L}$ standard before and after the trapping column insert; (e and f) BPA blank before and after the trapping column insert; ( $g$ and $h$ ) BPA in real sample before and after the trapping column insert. very time consuming, the trapping column was preferred to reduce the mentioned measurement uncertainty.

\section{Method performance}

The linear range of this method was $0.20-25 \mu \mathrm{g} / \mathrm{L}$ and the value of the coefficient correlation for the calibration curve was 0.996. The LOD and LOQ were 0.048 and $0.16 \mu \mathrm{g} / \mathrm{L}$, respectively. These results indicated that our method is more sensitive than those previously reported, which measured BPA without any derivation process. ${ }^{9,18,32-34}$ The recoveries $(\%)$ for BPA at three spiked levels $(0.50,1.0,10.0 \mu \mathrm{g} / \mathrm{L})$ were 82.2 , 88.9 and 85.1, respectively; further, the relative standard deviations (RSD, \%) were 15.9, 4.2 and 1.8, respectively. Carryover tests indicated that there was no BPA detected in all three blank samples, which implied that there were no BPA residues in trap column or analysis column. In addition, it is worth mentioning that the SPE method is widely accepted for sample preparation while being less time-consuming. In the present work, due to only a small amount of infant urine samples being analyzed, the liquid-liquid extraction method instead of SPE was used to reduce the routine cost. In summary, the method sensitivity, accuracy and precision indicated that the trapping column could effectively isolate any BPA interference in the mobile phase, which allows for a reliable determination of trace BPA in infant urine samples.

\section{BPA concentration in infant urine samples}

The second goal of the present work was preliminary to survey Chinese infant exposure to BPA. We determined BPA in 48 infant urine samples. The results showed that BPA was detectable in almost all subjects with a detection frequency of 93\%. The median level was 0.13 (average, 0.36) and ranged from $<\mathrm{LOD}$ to $5.04 \mu \mathrm{g} / \mathrm{L}$ in this small cohort. Due to the relatively low BPA levels in infant urine (Table 2), any very subtle background contamination may cause a big measurement uncertainty. Given that the trap column was not applied, the method background of BPA was at a level of $0.8 \mu \mathrm{g} / \mathrm{L}$, approximately. The LOD shall increase to $2.4 \mu \mathrm{g} / \mathrm{L}$ when it was defined as being three-times as high as the background; then, the LOQ shall increase to $8.0 \mu \mathrm{g} / \mathrm{L}$ when the LOQ is defined as 3.33 times of the LOD. Concerning this LOQ, only two subjects (i.e., $4.2 \%$ detectable frequency) shall be quantitatively assessed in this cohort. As a matter of fact, the median concentrations of BPA in Chinese infants are similar to the reported concentrations in Germany, Canada and USA. ${ }^{19-22}$ Thus the present work indicated that Chinese infants also broadly exposed to BPA.

The estimated daily BPA intake has decreased significantly from $2003-2004$ to $2011-2012,,^{17,22}$ but high levels of BPA can still be observed in some infant urine samples. ${ }^{18}$ The distinct exposure differences may be because of the different life style, age and regional different BPA usage. Although the BPA concentrations in the infants are generally lower than in the old

Table 2 BPA concentrations of infant urine samples in the current study and previous literature $(\mu \mathrm{g} / \mathrm{L})$

\begin{tabular}{|c|c|c|c|c|c|c|c|}
\hline Location & Age group & Detection frequency, $\%$ & Mean (Median) & Range & Sample time & LOD & LOQ \\
\hline Current Study & $<6$ months & 93 & $0.36(0.13)$ & $<\mathrm{LOD}-5.04$ & Mar. 2012 - Dec. 2014 & 0.048 & 0.16 \\
\hline Germany $^{20}$ & $1-5$ month & 42 & $(0.225)$ & $<$ LOD - 17.8 & Mar. - June 2008 & 0.15 & 0.45 \\
\hline Canada $^{19}$ & $<3$ months & 40 & $(0.2)$ & $<\mathrm{LOD}-9.4$ & Dec. 2009 - Dec. 2010 & 0.2 & - \\
\hline USA $^{21}$ & 7 - 44 days & 100 & $(0.38)$ & $0.21-0.79^{\mathrm{a}}$ & 2012 & 0.02 & 0.1 \\
\hline $\mathrm{USA}^{22}$ & $3-27$ days & 71 & $(0.2)^{\mathrm{a}}$ & $<\mathrm{LOD}-6.3^{\mathrm{a}}$ & Dec. 2012 - Aug. 2013 & - & 0.1 \\
\hline $\mathrm{USA}^{18}$ & $<4$ months & 93 & 0.96 & $0.5-2$ & Nov. 2006 - Aug. 2008 & 0.4 & - \\
\hline
\end{tabular}

a. Approximately. 
children and adults, $, 9,18,34,35$ because of their much greater susceptibility, the health risk needs to be investigated closely.

\section{Conclusions}

In this study, the HPLC mobile-phase interference of BPA was successfully isolated by hyphenating a short reversed-phase $\mathrm{C}_{18}$ column in between the LC solvent mixer and the autosampler. Because the whole procedure can be accomplished within $15 \mathrm{~min}$, it is still a high-throughput analysis method. The sensitivity, accuracy and precision indicated that this method is reliable for BPA measurements at trace levels. This method has also been validated by measurements of 48 infant urine samples. The findings of BPA in Chinese infants were comparable to BPA in other cohorts; the high detection frequency demonstrated that nearly all infants are exposed to BPA, the risks of which need to be evaluated in the large-scale epidemiological studies.

\section{Acknowledgements}

This work was supported by Zhejiang Provincial Natural Science Foundation (Grant No. LY14B070005), NSFC Research Foundation (Grant No. 21407144) and Fujian Nature Science of Foundation (Grand No. 2015J05038).

\section{Conflict of Interest}

The authors have declared no conflict interest.

\section{References}

1. D. Arenholt-Bindslev, V. Breinholt, A. Preiss, and G. Schmalz, Clin. Oral. Investig., 1999, 3, 120.

2. S. R. Howe, L. Borodinsky, and R. S. Lyon, J. Coating Technol., 1998, 70, 69.

3. J. Sajiki and J. Yonekubo, Chemosphere, 2003, 51, 55.

4. W. Dekant and W. Völkel, Toxicol. Appl. Pharmacol., 2008, 228, 114.

5. Y. B. Wetherill, B. T. Akingbemi, J. Kanno, J. A. Mclachlan, A. Nadal, C. Sonnenschein, C. S. Watson, R. T. Zoeller, and S. M. Belcher, Reprod. Toxicol., 2007, 24, 178.

6. M. Casas, D. Valvi, N. Luque, A. Ballesteros-Gomez, A. E. Carsin, M. F. Fernandez, H. M. Koch, M. A. Mendez, J. Sunyer, and S. Rubio, Environ. Int., 2013, 56, 10.

7. J. Chevrier, R. B. Gunier, A. Bradman, N. T. Holland, A. M. Calafat, B. Eskenazi, and K. G. Harley, Environ. Health Perspect., 2012, 121, 138.

8. A. L. Heffernan, L. L. Aylward, A. J. Samidurai, P. S. W. Davies, L. M. L. Toms, P. D. Sly, and J. F. Mueller, Environ. Int., 2014, 68C, 139.

9. T. H. Lassen, H. Frederiksen, T. K. Jensen, J. H. Petersen, U. N. Joensen, K. M. Main, N. E. Skakkebaek, A. Juul, N. Jørgensen, and A. M. Andersson, Environ. Health Perspect., 2014, 122, 478.

10. C. A. Snijder, D. Heederik, F. H. Pierik, A. Hofman, V. W. Jaddoe, H. M. Koch, M. P. Longnecker, and A. Burdorf, Environ. Health Perspect., 2013, 121, 393.

11. J. L. Coughlin, B. Winnik, and B. Buckley, Anal. Bioanal. Chem., 2011, 401, 995.
12. H. Frederiksen, L. Aksglaede, K. Sorensen, O. Nielsen, K. M. Main, N. E. Skakkebaek, A. Juul, and A. M. Andersson, Int. J. Hyg. Environ. Health, 2013, 216, 710.

13. S. X. Mei, S. D. Hui, W. B. Shuang, L. B. Bei, and H. F. Yuan, Biomed. Environ. Sci., 2014, 27, 471.

14. A. Myridakis, E. Balaska, C. Gkaitatzi, A. Kouvarakis, and E. G. Stephanou, Anal. Bioanal. Chem., 2015, 407, 2509.

15. X. Zhou, J. P. Kramer, A. M. Calafat, and X. Ye, J. Chromatogr. B, 2014, 944, 152.

16. H. Nakazawa, Y. Iwasaki, and R. Ito, Anal. Sci., 2014, 30, 25.

17. J. S. Lakind and D. Q. Naiman, Environ. Res., 2015, 142, 84.

18. K. Mendonca, R. Hauser, A. M. Calafat, T. E. Arbuckle, and S. M. Duty, Int. Arch. Occup. Environ. Health, 2014, 87, 13.

19. T. E. Arbuckle, L. Weiss, M. Fisher, R. Hauser, P. Dumas, R. Bérubé, A. Neisa, A. Leblanc, C. Lang, and P. Ayotte, Sci. Total. Environ., 2014, 508, 575.

20. W. V Lkel, M. Kiranoglu, and H. Fromme, Environ. Res., 2011, 111, 143.

21. R. M. Nachman, S. D. Fox, W. C. Golden, E. Sibinga, T. D. Veenstra, J. D. Groopman, and P. S. J. Lees, J. Pediatr., 2013, 162, 870.

22. R. M. Nachman, S. D. Fox, W. C. Golden, E. Sibinga, J. D. Groopman, and P. S. J. Lees, J. Pediatr., 2015, 167, 64.

23. S. D. Fox, R. T. Falk, T. D. Veenstra, and H. J. Issaq, J. Sep. Sci., 2011, 34, 1268.

24. WHO (World Health Organization), 2011, “Toxicological and Health Aspects of Bisphenol A", Report of Joint FAO/ WHO Expert Meeting, 2 - 5 November 2010, and Report of Stakeholder Meeting on Bisphenol A, 1 November 2010, http://whqlibdoc.who.int/publications/2011/97892141564274_ eng.pdf, accessed 4 January 2015.

25. C. Hong, Y. Wan, J. Naile, X. Zhang, S. Wiseman, M. Hecker, M. H. W. Lam, J. P. Giesy, and P. D. Jones, J. Chromatogr. A, 2010, 1217, 506.

26. G. Provencher, R. Bérubé, P. Dumas, J. F. Bienvenu, É. Gaudreau, P. Bélanger, and P. Ayotte, J. Chromatogr. A, 2014, 1348, 97.

27. L. Liu, J. She, X. Zhang, J. Zhang, M. Tian, Q. Huang, and H. Shen, J. Sep. Sci., 2014, 38, 247.

28. U.S. Department of Health and Human Services, Food and Drug Administration, Center for Drug Evaluation and Research (CDER), Center for Veterinary Medicine (CVM), "Guidance for Industry, Bioanalytical Method Validation", ed. May 2001.

29. L. Liu, T. Xia, X. Zhang, D. B. Barr, A. Alamdar, J. Zhang, M. Tian, Q. Huang, and H. Shen, Anal. Bioanal. Chem., 2014, 406, 5049 .

30. A. Kärrman, B. B. Van, U. Järnberg, L. Hardell, and G. Lindström, Anal. Chem., 2005, 77, 864.

31. A. G. Asimakopoulos and N. S. Thomaidis, J. Chromatogr. $B, \mathbf{2 0 1 5}, 1,85$.

32. A. M. Calafat, X. Ye, L. Y. Wong, J. A. Reidy, and L. L. Needham, Environ. Health Perspect., 2008, 116, 39.

33. W. Völkel, M. Kiranoglu, and H. Fromme, Toxicol. Lett., 2008, 179, 155.

34. F. Zhou, L. Zhang, A. Liu, S. Yang, J. Yuan, X. Yu, F. Xu, Q. Xu, and C. Cheng, J. Chromatogr. B, 2013, 938C, 80.

35. M. K. Morgan, P. A. Jones, A. M. Calafat, X. Ye, C. W. Croghan, J. C. Chuang, N. K. Wilson, M. S. Clifton, Z. Figueroa, and L. S. Sheldon, Environ. Sci. Technol., 2011, 45, 5309 . 